\title{
High versus Moderate Intensity Running Exercise to Impact Cardiometabolic Risk Factors: The Randomized Controlled RUSH-Study
}

\author{
Wolfgang Kemmler, ${ }^{1}$ Michael Scharf, ${ }^{2}$ Michael Lell, ${ }^{2}$ \\ Carina Petrasek, ${ }^{1}$ and Simon von Stengel ${ }^{1}$ \\ ${ }^{1}$ Institute of Medical Physics, Friedrich-Alexander University Erlangen-Nürnberg, Henkestrasse 91, 91052 Erlangen, Germany \\ ${ }^{2}$ Institute of Radiology, Friedrich-Alexander University Erlangen-Nürnberg, Maximiliansplatz 1, 91054 Erlangen, Germany \\ Correspondence should be addressed to Wolfgang Kemmler; wolfgang.kemmler@imp.uni-erlangen.de
}

Received 9 December 2013; Revised 11 January 2014; Accepted 30 January 2014; Published 11 March 2014

Academic Editor: Brad J. Schoenfeld

Copyright ( $) 2014$ Wolfgang Kemmler et al. This is an open access article distributed under the Creative Commons Attribution License, which permits unrestricted use, distribution, and reproduction in any medium, provided the original work is properly cited.

\begin{abstract}
Aerobic exercise positively impacts cardiometabolic risk factors and diseases; however, the most effective exercise training strategies have yet to be identified. To determine the effect of high intensity (interval) training (HI(I)T) versus moderate intensity continuous exercise (MICE) training on cardiometabolic risk factors and cardiorespiratory fitness we conducted a 16-week crossover RCT with partial blinding. Eighty-one healthy untrained middle-aged males were randomly assigned to two study arms: (1) a HI(I)Tgroup and (2) a sedentary control/MICE-group that started their MICE protocol after their control status. HI(I)T focused on interval training ( $90 \mathrm{sec}$ to $12 \mathrm{~min}>85-97.5 \% \mathrm{HRmax}$ ) intermitted by active recovery (1-3 min at $65-70 \% \mathrm{HRmax}$ ), while MICE consisted of continuous running at 65-75\% HRmax. Both exercise groups progressively performed 2-4 running sessions/week of 35 to $90 \mathrm{~min} /$ session; however, protocols were adjusted to attain similar total work (i.e., isocaloric conditions). With respect to cardiometabolic risk factors and cardiorespiratory fitness both exercise groups demonstrated similar significant positive effects on MetS-Z-Score (HI(I)T: $-2.06 \pm 1.31, P=.001$ versus MICE: $-1.60 \pm 1.77, P=.001$ ) and (relative) $\mathrm{VO}_{2} \max (\mathrm{HI}(\mathrm{I}) \mathrm{T}: 15.6 \pm 9.3 \%$, $P=.001$ versus MICE: $10.6 \pm 9.6 \%, P=.001$ ) compared with the sedentary control group. In conclusion, both exercise programs were comparably effective for improving cardiometabolic indices and cardiorespiratory fitness in untrained middle-aged males.
\end{abstract}

\section{Introduction}

Higher levels of cardiorespiratory fitness are associated with lower risk of all causes of mortality and cardiovascular/coronary heart diseases (CVD/CHD) [1]. Exercise significantly impacts cardiorespiratory fitness and is thus strongly recommended in both primary and secondary prevention of cardiometabolic diseases [2-4]. However, the most efficient exercise training strategies to positively affect cardiorespiratory fitness and cardiometabolic risk factors have yet to be identified. With respect to exercise intensity, there is some evidence that walking may be as effective as running for reducing cardiometabolic risk [5] at least when adjusting for energy expenditure (i.e., work). However, running is much more time efficient due to its higher physiological and biomechanical intensity [6]. This aspect is substantial, since lack of time was consistently reported as one of the central reasons for inactivity in Germany [7]. Developing this idea further, low volume/high intensity protocols should be a promising tool for impacting cardiometabolic risk in sedentary subjects. In fact, a number of corresponding studies that focus on prevention or rehabilitation of metabolic and cardiac diseases including myocardial infarction [8] applied low volume/high intensity (interval) training ("HI(I)T") [913]. Beside its apparent time effectiveness a main pro for HIIT is its significantly higher effect on $\mathrm{VO}_{2} \max$, the parameter considered as the typical marker of cardiorespiratory fitness [14], compared with "moderate intensity continuous exercise" (MICE) protocols traditionally applied in this research field 
(review in [15-19]). Since most of these studies focus on subjects with severe CAD and/or heart failure (review in: $[15-17,19,20])$ exercising on electronically braked cycle ergometers, it is doubtful, whether these data are transferable to the general population. Comparative studies that address primarily prevention of CVD/CHD are scarce $[9,13,21-24]$.

For that reason, the primary purpose of this randomized controlled trial is to compare the effects of two running exercise protocols (HIIT versus MICE) under the premise of comparable "energy consumption" on the Metabolic Syndrome (MetS) as a sensitive cluster of metabolic and cardiac risk factors in untrained middle-aged males.

The primary hypothesis was that HIIT is significantly superior for impacting the Metabolic Syndrome (MetS) ZScore and the number of criteria of the MetS in this cohort. The secondary hypothesis was that HIIT is significantly more effective for increasing cardiorespiratory fitness compared with MICE.

\section{Materials and Methods}

The Running Study and Heart (RUSH) Trial was a 16week randomized controlled crossover study with three study arms (two exercise, one waiting/control group) of untrained middle-aged males (Figure 1). The study was conducted by the Institute of Medical Physics, Friedrich-Alexander University Erlangen-Nürnberg (FAU), Germany, during April 2011 and July 2012. The primary study aim was to compare the effects of a high intensity (interval) training protocol $(\mathrm{HI}(\mathrm{I}) \mathrm{T})$ versus a moderate intensity continuous exercise protocol (MICE) on physical performance, metabolic, and cardiac parameters. The study protocol was approved by the ethics committee of the Friedrich-Alexander University (FAU) of Erlangen-Nürnberg (Ethikantrag 4463). All the study participants gave written informed consent. The study was registered under www.clinicaltrials.gov (NCT01406730).

2.1. Main Endpoints. The primary study endpoints of the present contribution were as follows.

(i) Metabolic Syndrome (MetS) Z-Score according to Johnson et al. [25].

(ii) Number of MetS parameters according to the National Cholesterol Education Programme Adult Treatment Panel III (NCEP ATP III) definition [26].

Secondary study endpoints were as follows.

(i) Maximum aerobic capacity $\left(\mathrm{VO}_{2} \mathrm{max}\right)$

(ii) Metabolic Syndrome criteria constituting the NCEP ATP III MetS criteria (i.e., waist circumference, mean arterial pressure (MAP), fasting glucose, triglycerides, and HDL-C).

2.2. Study Participants, Inclusion, and Exclusion Criteria. Study characteristic procedures of recruitment and the flow chart of the trial were described in more details in an earlier publication [27]. In summary (Figure 4), detailed announcements in local newspapers or on radio stations

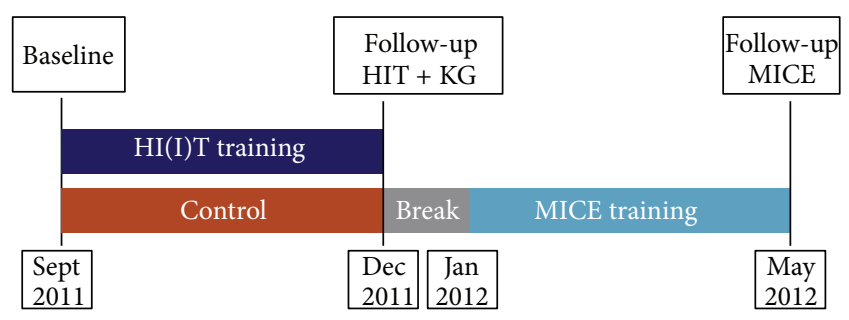

FIGURE 1: Time chart of the RUSH study.

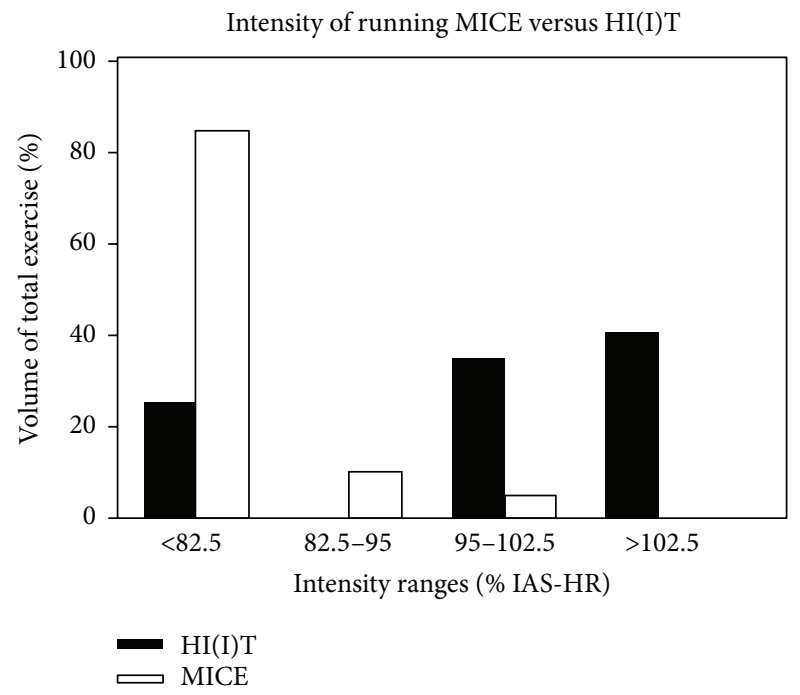

Figure 2: Exercise volume in different metabolic areas: HI(I)T versus MIC-running group. IAT: individual anaerobic threshold; HR: heart rate.

addressed untrained male subjects 30-50 years old. 121 subjects responded and were assessed for eligibility. Of these, 24 subjects had to be excluded due to the criteria of (a) male, $30-50$ years old ( $n=3$ ), (b) "untrained" (i.e., $\leq 1$ endurance exercise session/week; $\leq 2$ total exercise sessions/week during the last 2 years; $n=12$ ), (c) inflammatory diseases and pathological changes of the heart $(n=3)$, (d) medication/diseases affecting cardiovascular system, muscle, or joints/bone ( $n=1)$, (e) $<100$ Watt at ergometry $(n=0)$, (f) obesity (BMI $>35 \mathrm{~kg} / \mathrm{m}^{2} ; n=2$ ), and (g) more than 2 weeks of absence during the interventional period ( $n=$ 3). After detailed presentation of the study protocol, sixteen subjects were unwilling to join the randomization procedure and quit the study. Thus, finally 81 subjects were randomly assigned (computer generated block randomization ( $n=2-$ 4), stratified for age only) to two subgroups: (a) high intensity (interval) training ( $\mathrm{HI}(\mathrm{I}) \mathrm{T})$ group and (b) waiting-control group/moderate intensity continuous exercise (MICE) group, respectively, after crossover (Figures 1 and 4).

2.3. Measurements. Each participant was tested by the same researcher and at the same time of day $( \pm 1 \mathrm{~h})$. All assessments were determined in a blinded fashion. Researchers were not allowed to ask subjects about their exercise status (HIIT 


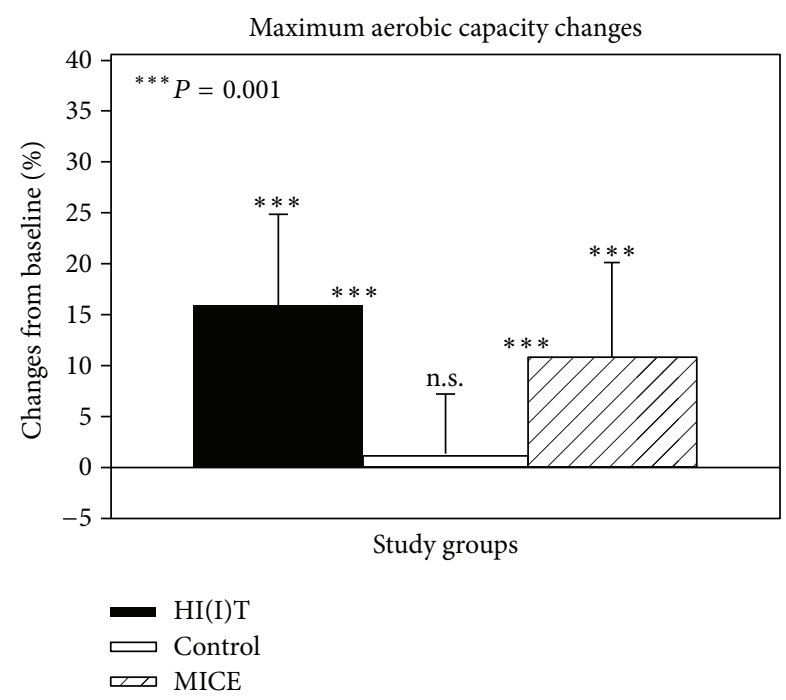

FIGURE 3: Percentage changes of relative $\mathrm{VO}_{2}$ max in the RUSH study groups including the inactive CG. Asterisk $(*)$ on the upper end of the error bar indicates significant changes within the group and asterisk between the bars indicates significant group differences. n.s. = nonsignificant.

or waiting CG/MICE). Each subject was provided with standardized rules for behaving prior to the tests in order to prevent confounding effects on primary and secondary endpoints (i.e., time to exhaustion (TTE) during stepwise treadmill test, blood pressure, and body composition).

2.3.1. Anthropometry. Height was determined with a stadiometer (Holtain, Crymych Dyfed, Great Britain). Weight, total, and regional body composition were determined using the bioimpedance technique (Inbody 230, Biospace, Seoul, Korea). Waist circumference was measured as the minimum circumference between the distal end of the rib cage and the top of the iliac crest along the midaxillary line.

2.3.2. Metabolic Syndrome Z-Score. MetS-Z-Score was calculated according to the formula proposed by Johnson et al. [25] based on the NCEP-ATP III criteria of the MetS [26]. According to these criteria five risk factors constituted the MetS: (1) raised triglyceride (TriGly) levels ( $\geq 150 \mathrm{mg} / \mathrm{dL}),(2)$ reduced HDL-C ( $<40 \mathrm{mg} / \mathrm{dL}$ for males), (3) raised blood pressure ( $\geq 130 / 85 \mathrm{mmHG}),(4)$ raised fasting plasma glucose $(\geq 100 \mathrm{mg} / \mathrm{dL}$ ), and (5) waist circumference (WC $\geq 102 \mathrm{~cm}$ for males).

Following Johnson et al. [25], the ATP-III cut-point for a male population and the corresponding baseline standard deviation (SD) of the entire RUSH-cohort were used for each parameter (i.e., HDL-C and triglycerides) of the individual data. In detail, the $Z$-Score was calculated using [ $(40-\mathrm{HDL}-$ C)/SD HDL-C $]+[($ TriGly -180$) /$ SD TriGly $]+[($ Glucose$100) /$ SD Glucose $]+[(\mathrm{WC}-100) / \mathrm{SD} W C]+[($ Mean arterial pressure (MAP) - 100)/SD MAP].
2.3.3. Blood Parameters. Blood was sampled from an antecubital vein in the morning (7:00-9:00 a.m.) after a $12 \mathrm{~h}$ overnight fast. Serum samples were centrifuged at $3000 \mathrm{RPM}$ for $20 \mathrm{~min}$. Total cholesterol, LDL-cholesterol, HDL-cholesterol, triglycerides, glucose, and uric acid (all: Olympus Diagnostica GmbH, Hamburg, Germany) were determined. During another visit, blood pressure was determined in a lying position after assessment of resting metabolic rate over $30 \mathrm{~min}$, using an automatic oscillometric device (Bosco; Bosch, Jungingen, Germany). Subjects refrained from coffee and/or tea for at least $12 \mathrm{~h}$ before testing and with more than $12 \mathrm{~h}$ passing since the last relevant physical exertion or exercise session.

\subsubsection{Aerobic Capacity $\left(\mathrm{VO}_{2} \max \right)$ and Running Economy.} During a stepwise treadmill test ( $3 \mathrm{~min} / 1 \mathrm{~km} / \mathrm{h}$ steps; $1^{\circ}$ slope) up to a voluntary maximum, $\mathrm{VO}_{2}$ was continuously determined breath by breath using an Oxycon mobile open spirometric system (Viasys, Conshohocken, PA, USA). For more details the reader is kindly referred to another publication [27]. Energy consumption/total work calculation according to the American College of Sports Medicine Guideline [28] is based also on $\mathrm{VO}_{2}$ and RER-assessment considering the individual aerobic threshold.

Running economy (RE) was assessed for the last $30 \mathrm{sec}$ of the second stage of the test (5:30 to 6:00 min), which was consistently kept below the anaerobic threshold [29].

2.4. Study Intervention. Figure 1 shows the design of the study intervention. For a detailed description of the intervention program the reader is referred to another publication [27]. Briefly, participants of the HI(I)T exercise group started on 16-week high intensity (running) exercise training in September 2011. After a short break around Christmas, participants of former control/waiting group started their 16-week moderate intensity continuous endurance exercise training in January 2012 and exercised until May 2012 (Figure 1). Each subject was provided with an individual training log that prescribed the intensity, volume, and frequency of the running exercise for the following 4 weeks. In both exercise groups, the weekly frequency of running exercise was progressively increased from 2 sessions/week at baseline to 3-4 sessions/week after week 8 . With respect to exercise intensity the exercise protocols were based on individual prescriptions of the heart rate (HR) in different metabolic areas which were determined by stepwise treadmill test to a voluntary maximum and calculated based on the individual aerobic threshold concept (IAT: minimum lactate $-2.0 \mathrm{mmol} / \mathrm{L}$ ) suggested by Dickhuth et al. [30]. The "Schwelle" Software (Schwelle Version 3, Bayreuth, Germany) was used to properly determine the IAT and the IAT-HR. In addition, the validity of the calculated IAT-HR was tested at baseline and after 8 weeks by running $30 \mathrm{~min}$ at this specification. Based on the subjects' perceived exertion ("too high/too low") IAT-HR was readjusted. In order to generate isocaloric condition and a comparable work load (in kcal) in both groups, running duration per exercise session was prescribed longer in the MICE group $(57 \pm 9$ versus $53 \pm$ 


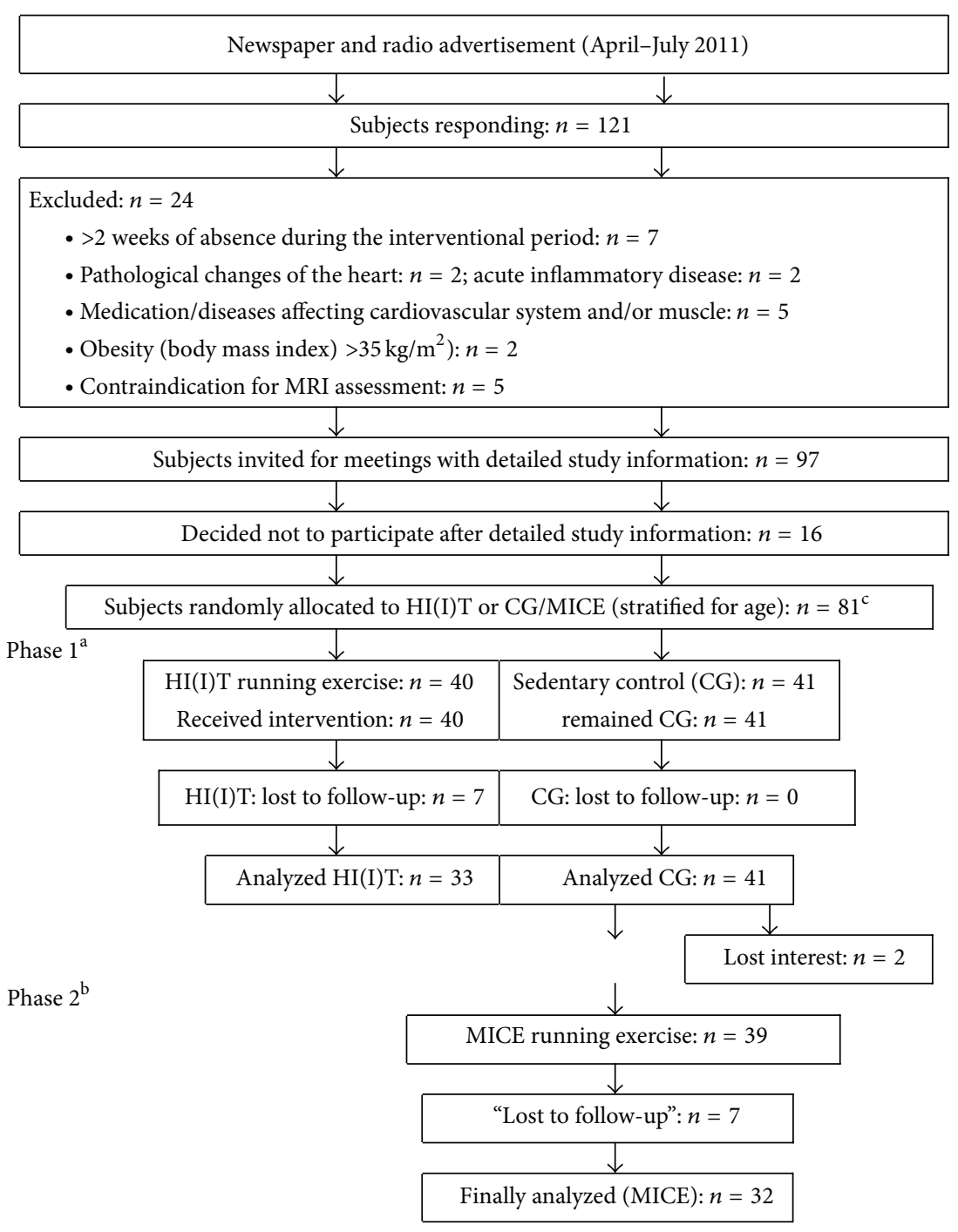

${ }^{\text {a }}$ September 2011 to December 2012 (Figure 1)

bJanuary 2012 to May 2012 (Figure1)

${ }^{\mathrm{c}} \mathrm{HI}(\mathrm{I}) \mathrm{T}$ : high intensity (interval) training; MICE: moderate intensity continuous exercise

FIGURE 4: Flow chart of the RUSH study.

$9 \mathrm{~min} / \mathrm{session}$ ) in order to compensate the higher intensity of the HI(I)T group [28].

Two sessions per week were consequently supervised by instructors who randomly checked exercise intensity by monitoring the HR watches (Polar RS 300, Kempele, Finland) which were provided for each participant.

2.4.1. HI(I)T Protocol. Figure 2 shows the rate of exercise volume in different metabolic areas for the HI(I)T and the MICE protocol. Briefly (core) exercise intensity during the intervention varied around $95 \%-110 \%$ of the IAT-HR (i.e., $>85$ to $97.5 \%$ HRmax) depending on the length of the interval ( $90 \mathrm{sec}-12 \mathrm{~min}$ ). Active rest periods of jogging or fast walking (1-3 min at 70-75\% IAT-HR, $665-70 \%$ HRmax) were prescribed between the intervals. Besides these intervals, continuous high intensity running sessions ranging from 25 to $45 \mathrm{~min}$ directly performed at the IAT ( $\approx 85 \% \mathrm{HRmax}$ ) were also part of the HI(I)T program. However $25 \%$ of total exercise volume of the HIIT group was performed at low exercise intensities (70-82.5\% IAT-HR) during warm-up, cool-down, and the active rest periods (Figure 2).

2.4.2. MICE Protocol. With slight exceptions (IAT-HR tests), exercise intensity of the MICE intervention was prescribed consistently low to moderate (70-82.5\% IAT-HR) (Figure 2). Duration of (continuous) running exercise was progressively increased during the 16 weeks of intervention and ranged from 35 to $90 \mathrm{~min}$ per session. 
2.5. Statistical Procedures. The sample size calculation was based on another study endpoint ("left ventricular enddiastolic volume") that is not presented here. Based on the sample size of 40 subjects per group and a Type 1 error of 5\%, the statistical power $(1-\beta)$ to detect a $5 \pm 7.5 \%$ difference between the groups was $85 \%$. All the subjects who took part in the follow-up measurements were included in the analysis ("finisher analysis").

Baseline and follow-up data are reported as mean values and standard deviations. Changes between baseline and follow-up in $\mathrm{HI}(\mathrm{I}) \mathrm{T}$ and MICE were reported as absolute (Table 2) and percentage changes (text, Figure 3 ). In addition, mean differences (with 95\% confidence intervals) between HI(I)T and MICE based on absolute changes are reported (Table 2). Differences of baseline characteristics were checked by one-way Anova. Distribution of the data was statistically and graphically checked. Nonnormally distributed parameters were log-transformed to obtain normally distributed data. Differences within groups were consistently analyzed by paired $t$-tests. Analyses of variance with repeated measurements consequently adjusted for baseline values were performed to check time group interactions. With respect to the comparison of all groups, post-hoc "Scheffé test" was also calculated (Figure 3). All tests were 2-tailed; statistical significance was accepted at $P<.05$. Effect sizes (ES) were calculated using Cohen's d. All statistical procedures were calculated with SPSS 21.0 (SPSS Inc., Chicago, IL) statistical procedures.

\section{Results}

Seven subjects of the HI(I)T (17.5\%) and nine subjects of the MICE group were lost to follow-up (21\%), while all the subjects of the CG $(n=41)$ were assessed. In this context, three subjects of the HI(I)T and four subjects of the MICE group quit the study due to injuries related to running exercise. One participant per group withdrew due to injuries related to the training protocol, two subjects of the HI(I)T and one subject of the MICE group due to injuries or diseases unrelated to exercise. Three subjects of the MICE and one subject of the HI(I)T group lost interest and cancelled their participation or did not start MICE after the control phase $(n=2)$.

Due to the comparable attendance rate of the HI(I)T $(40.5 \pm 5.4$ sessions; $82.6 \pm 11.1 \%)$ and MICE group (40.3 \pm 5.9 sessions; $82.3 \pm 12.0 \%)$ and the approach to compensate the higher exercise intensity of the HI(I)T group by higher exercise volume applied among the MICE group (2303 \pm 352 min versus $\mathrm{HI}(\mathrm{I}) \mathrm{T}: 2092 \pm 298 \mathrm{~min} ; P=.012$ ), total work was comparable between both groups (MICE: $30479 \pm 6566$ versus $\mathrm{HI}(\mathrm{I}) \mathrm{T}$ : $28966 \pm 5228 \mathrm{kcal}, P=.317)$.

Table 1 gives baseline characteristics of the subjects. With respect to these and other confounding factors (i.e., diet, lifestyle, exercise, medications, or diseases) that may have impacted the present results, no relevant differences at baseline (Table 1) or changes from baseline to follow-up were observed during study phase 1 or 2 (see Figure 4).
Weight significantly decreased in both exercise groups (HI(I)T: $-1.3 \pm 2.3 \mathrm{~kg}, P=.004$ versus MICE: $-2.5 \pm$ $2.4 \mathrm{~kg}, P=.001)$; however, the reduction was significantly higher $(P=.046)$ among the MICE participants. Significant weight gain $(1.2 \pm 2.3 \mathrm{~kg}, P=.002)$, resulting in significant differences $(P=.001)$ compared to MICE and HI(I)T, was observed for the CG. Lean body mass was maintained in the HI(I)T- $(0.4 \pm 2.1 \%, P=0.381)$ and CG $(0.7 \pm 2.3 \% ; P=0.072)$ but significantly dropped in the MICE group $(-1.1 \pm 1.8 \%$, $P=0.003)$. Body fat mass similarly $(P=.261)$ decreased in both exercise groups $(\mathrm{HI}(\mathrm{I}) \mathrm{T}:-4.9 \pm 9.0 \%, P=0.010$ versus MICE: $-9.5 \pm 10.4 \%, P<0.001)$ but increased in the CG $(3.4 \pm 9.1 \%, P=.012)$.

3.1. Study Endpoints. At baseline MetS-Z-Score and number of MetS parameters of the HI(I)T group were significantly higher compared with MICE and control (Table 2).

Metabolic Syndrome score according to Johnson et al. [25] significantly improved $(P=.001)$ in both exercise groups (HI(I)T: $-2.06 \pm 1.31$ versus MICE: $-1.60 \pm 1.77)$. Although changes of the control group were also significant $(-0.30 \pm 0.75, P=.0 .42)$ changes of both exercise groups were significantly more favorable $(P=.001)$ (Table 2$)$. At the same time, the number of MetS risk factors declined significantly $(P=.001)$ in both exercise groups (HI(I)T: $21.9 \pm 25.6 \%$ versus MICE: $43.8 \pm 31.5 \% ; P=.336)$ and was unchanged in the CG $(1.3 \pm 25.6, P=0.729)$. Changes of both exercise groups for this parameter significantly differed $(P=.001)$ from the CG $(3.5 \pm 17.9 \%, P=.432)$

Thus the primary hypothesis that HIIT is significantly more effective compared with MICE to impact the Metabolic Syndrome (MetS) Z-Score and the number of criteria of the MetS clearly has to be rejected.

With respect to underlying mechanisms, Table 2 shows changes of parameters that constituted the MetS according to the NCEP ATP III MetS definition. Waist circumference was significantly reduced in both exercise groups (HI(I)T: $-2.3 \pm 2.7 \%$ versus MICE: $-2.6 \pm 2.9 ; P=.720$ ), while a nonsignificant rise $(P=.083)$ was observed for the CG $(0.6 \pm 2.3 \% ; P=.001$ compared with both exercise groups). At the same time, significant reductions of MAP $(P=.001)$ were observed for both exercise groups (HI(I)T: $-4.9 \pm 4.0 \%$ versus MICE: $-5.9 \pm 4.2 ; P=.318)$ that significantly differed from the result of the CG $(0.3 \pm 4.4 \% ; P=.611)$.

After adjusting for baseline values, no intergroup differences were determined for triglycerides that declined in all groups (HI(I)T: $-19.7 \pm 24.8 \%, P=.001$ versus MICE: $-4.6 \pm$ $28.8 \%, P=.361$ versus CG: $-6.8 \pm 33.9 \%, P=.208)$. HDL-C also changed favorably and reached significance level for all groups (HI(I)T: $8.8 \pm 5.3 \%, P=.001$ versus MICE: $2.3 \pm 4.8 \%$, $P=.014$ versus CG: $2.9 \pm 4.3 \%, P=.001)$. However, changes between the HI(I)T and the MICE as well as between HI(I)T and CG were significant $(P=.001)$, while no difference was observed between MICE and CG $(P=.912)$. Furthermore, no group (HI(I)T: $-1.4 \pm 10.0 \%$ versus MICE: $-0.8 \pm 6.6 \%$ versus CG: $-3.5 \pm 16.7 \%)$ showed significant changes $(P<$ .212) of fasting glucose. Lastly, no between group differences were determined for this parameter $(P<.637)$. 
TABLE 1: Baseline characteristics of the RUSH study groups. No significant differences between the groups were determined.

\begin{tabular}{|c|c|c|c|}
\hline Variables & HI(I)T group & MICE group & CG \\
\hline Age (years) & $43.9 \pm 5.0$ & $42.9 \pm 5.1$ & $42.5 \pm 5.6$ \\
\hline Body height (centimeter) & $181.1 \pm 7.0$ & $181.6 \pm 5.3$ & $181.7 \pm 5.3$ \\
\hline Body weight (kilogram) & $91.5 \pm 14.0$ & $89.5 \pm 12.3$ & $89.8 \pm 15.5$ \\
\hline Overweight $\left(\mathrm{BMI}>25.0 \mathrm{~kg} / \mathrm{m}^{2}\right)(n)$ & $78 \%$ & $73 \%$ & $77 \%$ \\
\hline Fat mass $(\%)^{\mathrm{a}}$ & $25.5 \pm 5.7$ & $23.8 \pm 6.0$ & $23.9 \pm 6.1$ \\
\hline Physical activity (index) ${ }^{\mathrm{b}}$ & $2.8 \pm 1.4$ & $2.6 \pm 1.1$ & $2.6 \pm 1.1$ \\
\hline Occupational working time (hours/week) & $42.7 \pm 8.5$ & $44.8 \pm 5.4$ & $44.6 \pm 7.4$ \\
\hline $\mathrm{VO}_{2} \max \left(\mathrm{mL} / \mathrm{min} / \mathrm{kg}^{-1}\right)$ & $35.9 \pm 5.6$ & $39.5 \pm 5.5$ & $37.9 \pm 6.3$ \\
\hline Total exercise volume (minutes/week) & $28.8 \pm 32.1$ & $32.4 \pm 37.3$ & $32.6 \pm 37.5$ \\
\hline "Athletic" history $(n)^{c}$ & 31 & 29 & 29 \\
\hline Energy intake (kilocalorie/day) & $2595 \pm 738$ & $2737 \pm 592$ & $2580 \pm 616$ \\
\hline Fat/protein/carbohydrates (gram/day) & $96 / 104 / 305$ & $105 / 105 / 322$ & $96 / 104 / 297$ \\
\hline
\end{tabular}

${ }^{a}$ As assessed by bioimpedance analysis; ${ }^{\mathrm{b}}$ based on a scale from 1 (very low) to 7 (very high) according to a subjective assessment of professional, household, and recreational activities; ${ }^{c}$ formerly ( $13 \pm 5$ years ago) engaged in competitive sports with relevant demands on aerobic capacity (running, swimming, biking, triathlon, soccer, handball, and hockey); BMI: body mass index.

(Relative) $\mathrm{VO}_{2} \max \left(\mathrm{mL} * \mathrm{~min}^{-1} * \mathrm{~kg}^{-1}\right)$ significantly $(P=$ $.001)$ increased in both exercise groups (HI(I)T: $15.6 \pm 9.3 \%$ versus MICE: $10.6 \pm 9.6 \%)$ with no significant difference between HIIT and MICE $(P=.121)$. Relative $\mathrm{VO}_{2}$ max did not relevantly change in the CG $(1.1 \pm 6.4 \%, P=.263)$, thus, changes of both exercise groups significantly differed $(P=.001)$ from the control. Of interest, nonweight adjusted absolute $\mathrm{VO}_{2} \max \left(\mathrm{mL} / \mathrm{min}^{-1}\right)$ differed significantly $(P=$ $.002)$ between both exercise groups in favor of HIIT $(14.7 \pm$ $9.3 \%, P=.001$ versus MICE: $7.9 \pm 7.4 \%, P=.001)$; however due to the significantly more distinct reductions of weight (but not LBM) in MICE this finding disappeared after calculating relative $\mathrm{VO}_{2}$ max changes (Figure 3 ).

Although these results make it difficult to generate a clear statement, for reasons discussed below, we also reject the secondary hypothesis that HIIT was significantly more effective to increase "cardiorespiratory fitness" compared with MICE.

\section{Discussion}

Summarizing the results, this study failed to detect a superiority of a $\mathrm{HI}(\mathrm{I}) \mathrm{T}$ compared with a MICE protocol on the Metabolic Syndrome (MetS) as a sensitive cluster of metabolic and cardiac risk factors even under the premise of comparable total "work" (...or "total energy consumption") in both protocols (Table 2). However, compared with a nontraining control group both exercise protocols were highly effective for impacting the MetS, albeit by different mechanisms. While both protocols were similarly effective in reducing blood pressure and waist circumference, the effect of HIIT on blood lipids/lipoproteins was considerably higher compared with MICE. Three studies that either compared a high intensity interval $[9,21]$ or a high intensity continuous exercise protocol [25] with a MICE protocol while adjusting for energy consumption focused on the MetS cluster. In their pilot study with 22 subjects (52 \pm 4 yr.) suffering from MetS according to WHO [31], Tjønna et al. [13] compared a HIIT $(4 \times 4 \mathrm{~min}$ at $90-95 \%$ HRmax intermitted by $3 \mathrm{~min}$ of active rest at $70 \%$ HRmax) with a traditional continuous running exercise training (47 min at 70\% HRmax) performed 3times per week. After 16 weeks of exercise the HIIT subgroup showed a significantly more pronounced reduction of the number of subjects with MetS (HIIT: $-46 \%, P<.05$ versus MICE: $-37 \%(P=.13)$ and the number of MetS criteria $(-32 \%, P<.001$ versus $12 \%, P<.05)$ compared with MICE. However, applying continuous running exercise over 6 months for overweight subjects with "mild-to-moderate dyslipidemia” ( $n=86,40-65$ yr.) Johnson et al. [25] did not confirm this superiority of high intensity exercise (65$80 \% \mathrm{VO}_{2}$ peak; $128 \mathrm{~min}$ or $19 \mathrm{~km} /$ week) compared with MICE (40-55\% $\mathrm{VO}_{2}$ peak; $205 \mathrm{~min}$ or $19 \mathrm{~km} /$ week). In contrast, although nonsignificant, the authors reported more favorable changes of MetS- $Z$-Score and number of MetS criteria according to NCEP-ATP III among the MICE subgroup. However, with respect to the general effectiveness of these exercise protocols on MetS parameters, both MICE and $\mathrm{HI}(\mathrm{I}) \mathrm{T}$ significantly outperformed the corresponding control group(s). Earnest et al. [9] generally confirmed the data of Johnson et al. [25] in their comparative exercise trial with 42 overweighed 32-60-year-old males. After 6 weeks of exercise (3-4 $\times \approx 40 \mathrm{~min} /$ week with $50-70 \% \mathrm{VO}_{2} \mathrm{max}$ ) subjects were randomly allocated to further 6 weeks of continuous running exercise at $50-70 \% \mathrm{VO}_{2} \max$ or a HIIT-protocol including up to 8 cycles of $2 \mathrm{~min}$ at $90-95 \% \mathrm{VO}_{2} \max$ intermitted by 2 min of active recovery $\left(50 \% \mathrm{VO}_{2} \mathrm{max}\right)$. MetS- $Z$-Score according to the American Heart Association [32] and the number of MetS-risk factors (HIIT: $-1.14 \pm 1.15$ versus MICE: $-1.03 \pm 1.68)$ significantly improved in both groups similarly. However, in a subgroup of persons at lower risk for insulin resistance (i.e., low HOMA-IR) only HIIT showed significant results. With respect to aerobic capacity/endurance performance both groups similarly and significantly improved (MICE: 3.1 versus HIIT: $2.9 \mathrm{~mL} * \mathrm{~min}^{-1} * \mathrm{~kg}^{-1}$ or 2.8 versus 3.1 min during treadmill test). Of relevance, since body mass 
TABLE 2: Effects of high intensity (interval) training (HI(I)T) versus moderate intensity continuous exercise (MICE) on Metabolic Syndrome parameters. Intergroup differences were adjusted for baseline values.

\begin{tabular}{|c|c|c|c|c|c|}
\hline & $\mathrm{HI}(\mathrm{I}) \mathrm{T}^{\mathrm{a}}$ & $\mathrm{MICE}^{\mathrm{a}}$ & $\begin{array}{c}\text { Mean difference } \\
95 \% \mathrm{CI}\end{array}$ & $P$ & $E S^{b}(d)$ \\
\hline \multicolumn{6}{|c|}{ Metabolic Syndrome index ( $Z$-Score) } \\
\hline Before & $0.95 \pm 3.36$ & $-1.82 \pm 2.53$ & - & .001 & \\
\hline After & $-1.11 \pm 3.46$ & $-3.42 \pm 2.88$ & - & - & \\
\hline Difference & $-2.06 \pm 1.31^{* * * a}$ & $-1.60 \pm 1.77^{* * * a}$ & $0.46(1.07$ to -0.22$)$ & .291 & 0.29 \\
\hline \multicolumn{6}{|c|}{$\begin{array}{l}\text { Numbers of risk factors of the } \\
\text { Metabolic Syndrome ( } n \text { out of } 5 \text { ) }\end{array}$} \\
\hline Before & $2.51 \pm 1.29$ & $1.60 \pm 1.13$ & - & .005 & \\
\hline After & $1.97 \pm 1.20$ & $0.90 \pm 1.09$ & - & - & \\
\hline Difference & $-0.55 \pm 0.62^{* * *}$ & $-0.70 \pm 0.59^{* * *}$ & 0.15 ( -0.16 to 0.46$)$ & .336 & 0.25 \\
\hline \multicolumn{6}{|c|}{ Waist circumference $(\mathrm{cm})$} \\
\hline Before & $101.5 \pm 9.0$ & $98.0 \pm 9.5$ & - & .129 & \\
\hline After & $99.2 \pm 9.2$ & $95.4 \pm 9.3$ & - & - & \\
\hline Difference & $-2.3 \pm 2.8^{* * *}$ & $-2.6 \pm 3.0^{* * *}$ & $0.26(-1.18$ to 1.70$)$ & .720 & 0.10 \\
\hline \multicolumn{6}{|c|}{$\begin{array}{l}\text { Mean arterial pressure (MAP) } \\
(\mathrm{mm} / \mathrm{Hg})\end{array}$} \\
\hline Before & $111.5 \pm 10.1$ & $104.7 \pm 7.1$ & - & .003 & \\
\hline After & $106.6 \pm 8.9$ & $98.8 \pm 7.2$ & - & - & \\
\hline Difference & $-4.9 \pm 4.0^{* * *}$ & $-5.9 \pm 4.2^{* * *}$ & $1.03(-1.01$ to 3.06$)$ & .318 & 0.24 \\
\hline \multicolumn{6}{|c|}{ Triglycerides $(\mathrm{mg} / \mathrm{dL})$} \\
\hline Before & $166.3 \pm 85.1$ & $127.0 \pm 55.2$ & - & .013 & \\
\hline After & $146.5 \pm 82.2$ & $122.4 \pm 59.4$ & - & - & \\
\hline Difference & $-19.7 \pm 26.8^{* * *}$ & $-4.6 \pm 28.8^{\text {n.s. }}$ & 15.1 (2.0 to 28.2$)$ & .083 & 0.54 \\
\hline \multicolumn{6}{|c|}{$\begin{array}{l}\text { High density lipoprotein Cholesterol } \\
(\mathrm{mg} / \mathrm{dL})\end{array}$} \\
\hline Before & $43.1 \pm 12.1$ & $50.4 \pm 10.7$ & - & .001 & \\
\hline After & $51.9 \pm 12.7$ & $52.7 \pm 10.4$ & - & - & \\
\hline Difference & $8.8 \pm 5.3^{* * *}$ & $2.3 \pm 4.8^{*}$ & 6.46 (3.88 to 9.05$)$ & .001 & 1.29 \\
\hline \multicolumn{6}{|c|}{ Glucose (mg/dL) } \\
\hline Before & $92.2 \pm 11.3$ & $89.6 \pm 8.3$ & - & .258 & \\
\hline After & $90.7 \pm 7.6$ & $88.8 \pm 8.8$ & - & - & \\
\hline Difference & $-1.5 \pm 10.0^{\text {n.s. }}$ & $-0.8 \pm 6.6^{\text {n.s. }}$ & $0.76(5.18$ to -3.46$)$ & .693 & 0.008 \\
\hline
\end{tabular}

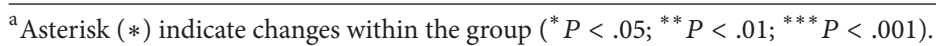

${ }^{\mathrm{b}}$ ES: effect size; 0.2: small; 0.5: moderate; 0.8: large.

decreased in both groups $(-1.3$ versus $-2.3 \mathrm{~kg})$, absolute $\mathrm{VO}_{2}$ max per se was not significantly affected $(0.25$ versus $0.13 \mathrm{~L} * \mathrm{~min}^{-1}$ ) by the running protocols.

Revisiting the issue of whether some cardiometabolic MetS parameters are more sensitive to HIIT or to MICE, one might think that the large number of comparative trials, reviews, and meta-analysis that focus on this topic (e.g., [17, 33-37]) should allow a meaningful conclusion. However, in view of the large variety of confounders in these studies (e.g., sex, age, initial levels, health and fitness status, prescribed medication, and type of exercise) that may impact the effects of exercise on cardiometabolic risk factors, it is difficult to draw general conclusions. Also problematic for an adequate interpretation, most dose-response or comparative studies compared continuous (not interval) high intensity endurance protocols that did not reach intensities (far) above the aerobic threshold [38]. Some of these studies that classified "moderate" or "vigorous" intensity at the lower end of the ACSM classification [33] (i.e., 3-6 MetS for moderate and 68 for vigorous exercise intensity) prescribed "high intensity workouts" that hardly exceed the exercise intensity of the MICE group in this study [36]. Taking these limitations into account and simply summarizing the data of present comparative studies, we are unable to detect a consistent superiority of HIIT versus MICE protocols (or vice versa) on cardiometabolic risk factors related to the MetS, independent of health, overweight, and fitness status $[9,13,17,22,23,39$ $42]$, although there are indeed some studies that reported more positive effects on single MetS risk factors for MICE or HIIT (e.g., [23, 24, 43]). 
With respect to "cardiorespiratory fitness", a strong tendency for higher $\mathrm{VO}_{2}$ max changes generated by the HIIT protocol of the present study was observed, which is mainly confirmed by comparative trials with untrained subjects with MetS or CHD/CVD [16, 17, 19, 20]. However, it is debatable whether higher $\mathrm{VO}_{2}$ max changes actually represented higher changes of cardiorespiratory fitness. In fact, this and other studies (e.g., [9]) observed even slightly higher changes of endurance performance ( speed $_{\max }$, time to exhaustion) by their MICE protocols, although $\mathrm{VO}_{2}$ max changes may be more distinct in the $\mathrm{HI}(\mathrm{I}) \mathrm{T}$ group. At first sight this result may appear contradictory, since $\mathrm{VO}_{2} \max$ is an important predictor of endurance capacity [44]. However, it is not the only determinant of this complex topic $[45,46]$. In fact, changes of running economy, another central determinant of endurance capacity, were significantly higher in our MICE group, which may account for the similar changes of endurance capacity. Thus, generating a comparable effect on endurance performance per se (i.e., TTE) the mechanism of HIIT versus MICE widely differs with respect to maximization $\left(\mathrm{VO}_{2} \mathrm{max}\right)$ and economization (RE). Hence, although we think that $\mathrm{VO}_{2}$ max may in general be a valid characteristic for cardiorespiratory fitness, a conclusion whether or not HIIT protocols are superior for impacting cardiorespiratory fitness should not be based solely on this parameter.

Some limitations and features of the RUSH study may complicate a direct comparison with most HIIT protocols cited above: (1) due to the study protocol the exercise groups were not evaluated in parallel but consecutively (Figure 1). Thus, seasonable changes of physical activity or diet may have impacted our results, although no corresponding changes were detected by the follow-up questionnaires. (2) The study protocol prescribed a combination of HIT and HI(I)T with intervals of $90 \mathrm{sec}$ to $12 \mathrm{~min}$ but also continuous bouts (25$40 \mathrm{~min}$ ) at the IAT. Although around or above the aerobic threshold, this approach differs from purebred "HI(I)T" protocols defined as repeated very short $(<45 \mathrm{sec})$ or short (2-4 min) bouts of high to near maximum intensity exercise [47]. (3) Although on the majority overweight, only in $33 \%$ of the RUSH participants the MetS was prevalent. Correspondingly, the average numbers of MetS criteria per subject were rather low $(2.0 \pm 1.2)$, which may prevent a more distinct reduction of this parameter. Therefore the authors opt for the use of the MetS- $Z$-Score, a continuous score that is based on individual subjects (raw) data, which may be more appropriate to properly assess the effect of the study intervention [25]. (4) The drop-out rates in both exercise groups were rather high $(\approx 20 \%)$. Independent of the exercise intensity, half of these subjects cited orthopedic problems for their withdrawal. Furthermore, a slight majority (HIIT: $n=16$ versus MICE: $n=15$ ) of the remaining males reported musculoskeletal problems and complaints longer than 7 days. These adverse effects may be related to the high body weight in this cohort and the abruptly increased mechanical impact due to the unaccustomed running exercise and the speedy progression of the running program. (5) With respect to exercise (intensity) compliance the authors did not analyze all the heart rate watches after the session but randomly selected 15-20 subjects per session (i.e., 50\%) for this procedure.
(6) In order to adequately focus on the effect of exercise intensity, unlike most other studies with healthy untrained persons, comparable work load for both groups was prescribed. However, the authors are aware that time effectiveness is an important benefit of HIIT protocols that largely account for its attractiveness [48].

In summary, both protocols favorably impacted cardiometabolic risk factors; however the superiority of HIIT protocols for positively impacting MetS as a cluster of relevant metabolic and cardiac risk factors cannot be confirmed, at least for this cohort of untrained middle-aged males. Furthermore, although $\mathrm{VO}_{2}$ max changes were significantly higher in the HI(I)T group, based on the slightly higher MICE effect on endurance capacity, we do not share the enthusiasm of other researchers in recommending pure HIIT protocols for increasing "cardiorespiratory fitness". We would rather favor a skilled combination of higher and lower exercise intensity (and volume) that may optimally affect performance parameters related to both economy and maximization.

\section{Conclusion}

Addressing the strengths and limitations of HIIT protocols in the field of primary and secondary prevention, time efficiency along with effectiveness is a clear pro for HIIT, taking into account the sedentary lifestyle and low affinity to exercise of most middle-aged subjects [7]. At the same time, there is some evidence that HIIT was perceived as "more enjoyable" compared with the more monotone MICE [49] another pro that may increase exercise adherence. One may argue that the rather high intensity of HIIT may provoke cardiovascular complications during exercise at least in subjects with cardiovascular diseases. However, although the statistical power of this study may be still ( $\approx 176.000$ exercise training hours at all) too low to detect differences between groups, a recent study of Rognmo et al. [12] listed extremely low rates of CHD events for both methods HIIT and MICE.

Thus, although we cannot confirm the general superiority of HIIT, with respect to efficiency and adherence, we strongly recommend HIIT as a reasonable component of endurance exercise protocols for prevention and rehabilitation of cardiometabolic diseases.

\section{Conflict of Interests}

The authors declare that there is no conflict of interests regarding the publication of this paper.

\section{Acknowledgments}

The authors acknowledge support of the Institute of Radiology, the Institute of Sport Sciences, FAU-Erlangen-Nürnberg, and Nürnberg Hospital.

\section{References}

[1] S. Kodama, K. Saito, S. Tanaka et al., "Cardiorespiratory fitness as a quantitative predictor of all-cause mortality and cardiovascular events in healthy men and women: a meta-analysis," 
Journal of the American Medical Association, vol. 301, no. 19, pp. 2024-2035, 2009.

[2] T. Kavanagh, "Exercise in the primary prevention of coronary artery disease," The Canadian Journal of Cardiology, vol. 17, no. 2, pp. 155-161, 2001.

[3] C. J. Lavie, R. J. Thomas, R. W. Squires, T. G. Allison, and R. V. Milani, "Exercise training and cardiac rehabilitation in primary and secondary prevention of coronary heart disease," Mayo Clinic Proceedings, vol. 84, no. 4, pp. 373-383, 2009.

[4] D. L. Swift, C. J. Lavie, N. M. Johannsen et al., "Physical activity, cardiorespiratory fitness, and exercise training in primary and secondary coronary prevention," Circulation Journal, vol. 77, no. 2, pp. 281-292, 2013.

[5] P. T. Williams and P. D. Thompson, "Walking versus running for hypertension, cholesterol, and diabetes mellitus risk reduction," Arteriosclerosis, Thrombosis, and Vascular Biology, vol. 33, no. 5, pp. 1085-1091, 2013.

[6] L. D. Wilkin, A. Cheryl, and B. L. Haddock, "Energy expenditure comparison between walking and running in average fitness individuals," Journal of Strength and Conditioning Research, vol. 26, no. 4, pp. 1039-1044, 2012.

[7] A. Rütten, K. Abu-Omar, R. Meierjürgen, A. Lutz, and W. Adlwarth, "Was bewegt die Nicht-Beweger?" Prävention und Gesundheitsförderung, vol. 4, pp. 245-250, 2009.

[8] H. Adachi, A. Koike, T. Obayashi et al., "Does appropriate endurance exercise training improve cardiac function in patients with prior myocardial infarction?" European Heart Journal, vol. 17, no. 10, pp. 1511-1521, 1996.

[9] C. P. Earnest, M. Lupo, J. Thibodaux et al., "Interval training in men at risk for insulin resistance," International Journal of Sports Medicine, vol. 34, no. 4, pp. 355-363, 2013.

[10] M. J. Gibala, "High-intensity interval training: a time-efficient strategy for health promotion?" Current Sports Medicine Reports, vol. 6, no. 4, pp. 211-213, 2007.

[11] T. Moholdt, U. Wisløff, T. I. L. Nilsen, and S. A. Slørdahl, "Physical activity and mortality in men and women with coronary heart disease: a prospective population-based cohort study in Norway (the HUNT study)," European Journal of Cardiovascular Prevention and Rehabilitation, vol. 15, no. 6, pp. 639-645, 2008.

[12] O. Rognmo, T. Moholdt, H. Bakken et al., "Cardiovascular risk of high- versus moderate-intensity aerobic exercise in coronary heart disease patients," Circulation, vol. 126, no. 12, pp. 14361440, 2012.

[13] A. E. Tjønna, S. J. Lee, Ø. Rognmo et al., "Aerobic interval training versus continuous moderate exercise as a treatment for the metabolic syndrome: a pilot study," Circulation, vol. 118, no. 4, pp. 346-354, 2008.

[14] L. Dalleck and A. Dalleck, "The ACSM exercise intensity guidelines for cardiorespiratory fitness: why the misuse?" Journal of Exercise Physiology Online, vol. 11, no. 4, pp. 1-11, 2008.

[15] T. Guiraud, A. Nigam, V. Gremeaux et al., "High-intensity interval training in cardiac rehabilitation," Sports Medicine, vol. 42, no. 7, pp. 587-605, 2012.

[16] M. J. Haykowsky, M. P. Timmons, C. Kruger et al., "Metaanalysis of aerobic interval training on exercise capacity and systolic function in patients with heart failure and reduced ejection fractions," The American Journal of Cardiology, vol. 111, no. 10, pp. 1466-1469, 2013.

[17] C.-L. Hwang, Y.-T. Wu, and C.-H. Chou, "Effect of aerobic interval training on exercise capacity and metabolic risk factors in people with cardiometabolic disorders: a meta-analysis," Journal of cardiopulmonary rehabilitation and prevention, vol. 31, no. 6, pp. 378-385, 2011.

[18] O. J. Kemi and U. Wisløff, "High-intensity aerobic exercise training improves the heart in health and disease," Journal of Cardiopulmonary Rehabilitation and Prevention, vol. 30, no. 1, pp. 2-11, 2010.

[19] K. S. Weston, U. Wisloff, and J. S. Coombes, "High-intensity interval training in patients with lifestyle-induced cardiometabolic disease: a systematic review and meta-analysis," British Journal of Sports Medicine, 2013.

[20] P. Meyer, M. Gayda, M. Juneau et al., "High-intensity aerobic interval exercise in chronic heart failure," Current Heart Failure Reports, vol. 10, no. 2, pp. 130-138, 2013.

[21] L. DiPietro, J. Dziura, C. W. Yeckel, and P. D. Neufer, "Exercise and improved insulin sensitivity in older women: evidence of the enduring benefits of higher intensity training," Journal of Applied Physiology, vol. 100, no. 1, pp. 142-149, 2006.

[22] L. Nybo, E. Sundstrup, M. D. Jakobsen et al., "High-intensity training versus traditional exercise interventions for promoting health," Medicine and Science in Sports and Exercise, vol. 42, no. 10, pp. 1951-1958, 2010.

[23] G. O’Donovan, A. Owen, S. R. Bird et al., "Changes in cardiorespiratory fitness and coronary heart disease risk factors following 24 wk of moderate- or high-intensity exercise of equal energy cost," Journal of Applied Physiology, vol. 98, no. 5, pp. 1619-1625, 2005.

[24] K. Wallman, L. A. Plant, B. Rakimov, and A. J. Maiorana, "The effects of two modes of exercise on aerobic fitness and fat mass in an overweight population," Research in Sports Medicine, vol. 17, no. 3, pp. 156-170, 2009.

[25] J. L. Johnson, C. A. Slentz, J. A. Houmard et al., "Exercise training amount and intensity effects on metabolic syndrome (from studies of a targeted risk reduction intervention through defined exercise)," American Journal of Cardiology, vol. 100, no. 12, pp. 1759-1766, 2007.

[26] J. I. Cleeman, "Executive summary of the third report of the National Cholesterol Education Program (NCEP) expert panel on detection, evaluation, and treatment of high blood cholesterol in adults (adult treatment panel III)," Journal of the American Medical Association, vol. 285, no. 19, pp. 2486-2497, 2001.

[27] W. Kemmler and S. von Stengel, "Effects of high versus moderate intensity running exercise on aerobic performance," Journal of Strength and Conditioning Research. In press.

[28] ACSM, ACSM'S Guidelines for Exercise Testing and Prescription, Lippincott Williams \& Wilkins, Philadelphia, Pa, USA, 2000.

[29] J. Helgerud, "Maximal oxygen uptake, anaerobic threshold and running economy in women and men with similar performances level in marathons," European Journal of Applied Physiology and Occupational Physiology, vol. 68, no. 2, pp. 155$161,1994$.

[30] H. H. Dickhuth, M. Huonker, T. Münzel et al., "Individual anaerobic threshold for evaluation of competitive athletes and patients with left ventricular dysfunction," in Advances in Ergometry, T. G. Bachl and H. Löllgen, Eds., pp. 173-179, Springer, New York, NY, USA, 1991.

[31] WHO, "Definition of the metabolic syndrome, diagnosis, and classification of diabetes mellitus and its complication," Report of a WHO Consultation, World Health Organisation, Generva, Switzerland, 1999. 
[32] S. M. Grundy, J. I. Cleeman, S. R. Daniels et al., "Diagnosis and management of the metabolic syndrome: an American Heart Association/National Heart, Lung, and Blood Institute scientific statement," Circulation, vol. 112, no. 17, pp. 2735-2752, 2005.

[33] C. E. Garber, B. Blissmer, M. R. Deschenes et al., "Quantity and quality of exercise for developing and maintaining cardiorespiratory, musculoskeletal, and neuromotor fitness in apparently healthy adults: guidance for prescribing exercise," Medicine and Science in Sports and Exercise, vol. 43, no. 7, pp. 1334-1359, 2011.

[34] D. Hansen, P. Dendale, L. J. C. Van Loon, and R. Meeusen, “The impact of training modalities on the clinical benefits of exercise intervention in patients with cardiovascular disease risk or type 2 diabetes mellitus," Sports Medicine, vol. 40, no. 11, pp. 921-940, 2010.

[35] S. Kodama, S. Tanaka, K. Saito et al., "Effect of aerobic exercise training on serum levels of high-density lipoprotein cholesterol: a meta-analysis," Archives of Internal Medicine, vol. 167, no. 10, pp. 999-1008, 2007.

[36] D. P. Swain and B. A. Franklin, "Comparison of cardioprotective benefits of vigorous versus moderate intensity aerobic exercise," American Journal of Cardiology, vol. 97, no. 1, pp. 141-147, 2006.

[37] D. Vissers, W. Hens, J. Taeymans et al., "The effect of exercise on visceral adipose tissue in overweight adults: a systematic review and meta-analysis," PLoS ONE, vol. 8, no. 2, Article ID e56415, 2013.

[38] P. B. Laursen and D. G. Jenkins, "The scientific basis for highintensity interval training: optimising training programmes and maximising performance in highly trained endurance athletes," Sports Medicine, vol. 32, no. 1, pp. 53-73, 2002.

[39] T. C. Fu, C. H. Wang, P. S. Lin et al., "Aerobic interval training improves oxygen uptake efficiency by enhancing cerebral and muscular hemodynamics in patients with heart failure," International Journal of Cardiology, vol. 167, no. 1, pp. 41-50, 2013.

[40] F. Iellamo, V. Manzi, G. Caminiti et al., "Matched dose interval and continuous exercise training induce similar cardiorespiratory and metabolic adaptations in patients with heart failure," International Journal of Cardiology, vol. 167, no. 6, pp. 25612565, 2013.

[41] M. M. Moreira, H. P. C. De Souza, P. A. Schwingel, C. K. C. De Sá, and C. C. Zoppi, "Effects of aerobic and anaerobic exercise on cardiac risk variables in overweight adults," Arquivos Brasileiros de Cardiologia, vol. 91, no. 4, pp. 219-226, 2008.

[42] T. R. Thomas, S. B. Adeniran, and G. L. Etheridge, "Effects of different running programs on $\mathrm{VO}_{2}$ max, percent fat, and plasma lipids," Canadian journal of applied sport sciences. Journal canadien des sciences appliquees au sport, vol. 9, no. 2, pp. 55-62, 1984.

[43] U. Wisløff, A. Støylen, J. P. Loennechen et al., "Superior cardiovascular effect of aerobic interval training versus moderate continuous training in heart failure patients: a randomized study," Circulation, vol. 115, no. 24, pp. 3086-3094, 2007.

[44] A. W. Midgley, L. R. McNaughton, and M. Wilkinson, "Is there an optimal training intensity for enhancing the maximal oxygen uptake of distance runners? Empirical research findings, current opinions, physiological rationale and practical recommendations," Sports Medicine, vol. 36, no. 2, pp. 117-132, 2006.

[45] D. L. Costill, H. Thomason, and E. Roberts, "Fractional utilization of the aerobic capacity during distance running," Medicine and Science in Sports and Exercise, vol. 5, no. 4, pp. 248-252, 1973.

[46] P. E. Di Pramero, G. Atchou, J.-C. Bruckner, and C. Moia, "The energetics of endurance running," European Journal of Applied
Physiology and Occupational Physiology, vol. 55, no. 3, pp. 259266, 1986.

[47] M. Buchheit and P. B. Laursen, "High-intensity interval training, solutions to the programming puzzle: part II: anaerobic energy, neuromuscular load and practical applications," Sports Medicine, vol. 43, no. 10, pp. 927-954, 2013.

[48] M. J. Gibala, J. P. Little, M. J. Macdonald, and J. A. Hawley, "Physiological adaptations to low-volume, high-intensity interval training in health and disease," Journal of Physiology, vol. 590, no. 5, pp. 1077-1084, 2012.

[49] J. D. Bartlett, G. L. Close, D. P. M. Maclaren, W. Gregson, B. Drust, and J. P. Morton, "High-intensity interval running is perceived to be more enjoyable than moderate-intensity continuous exercise: implications for exercise adherence," Journal of Sports Sciences, vol. 29, no. 6, pp. 547-553, 2011. 


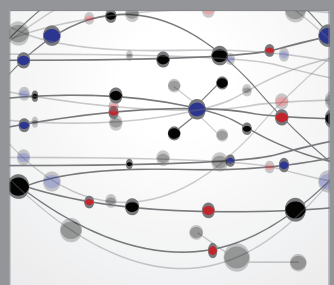

The Scientific World Journal
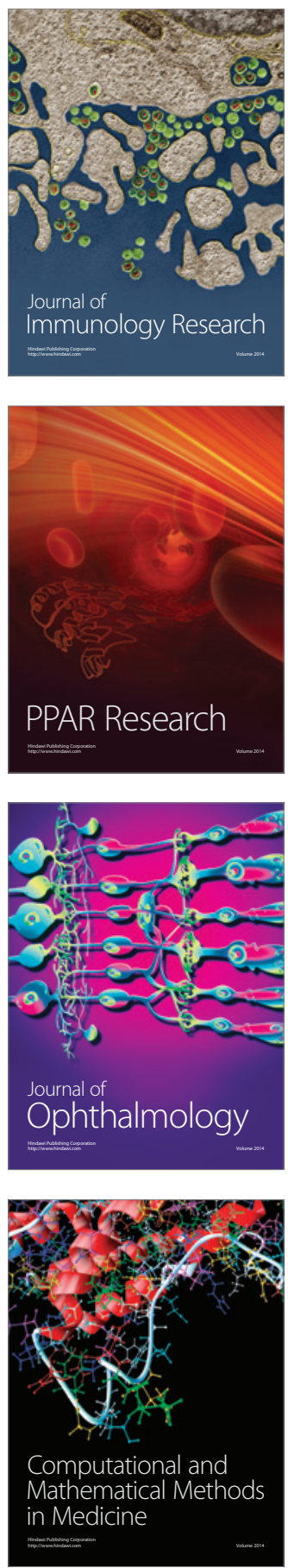

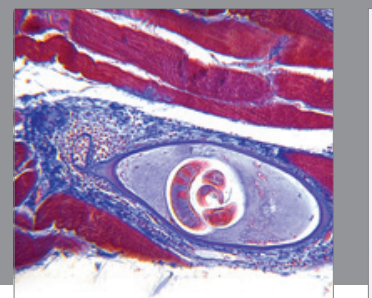

Gastroenterology

Research and Practice
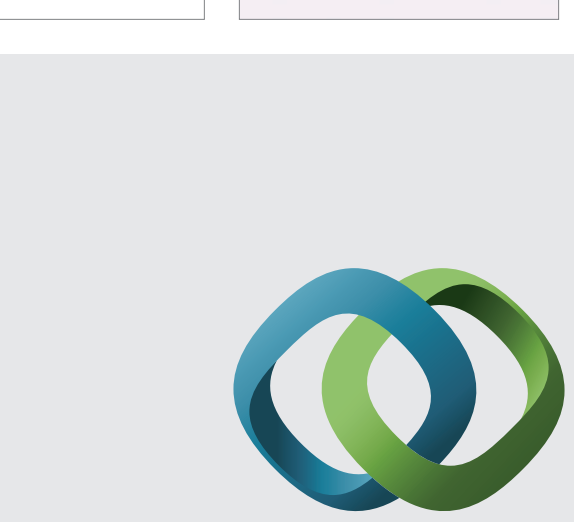

\section{Hindawi}

Submit your manuscripts at

http://www.hindawi.com
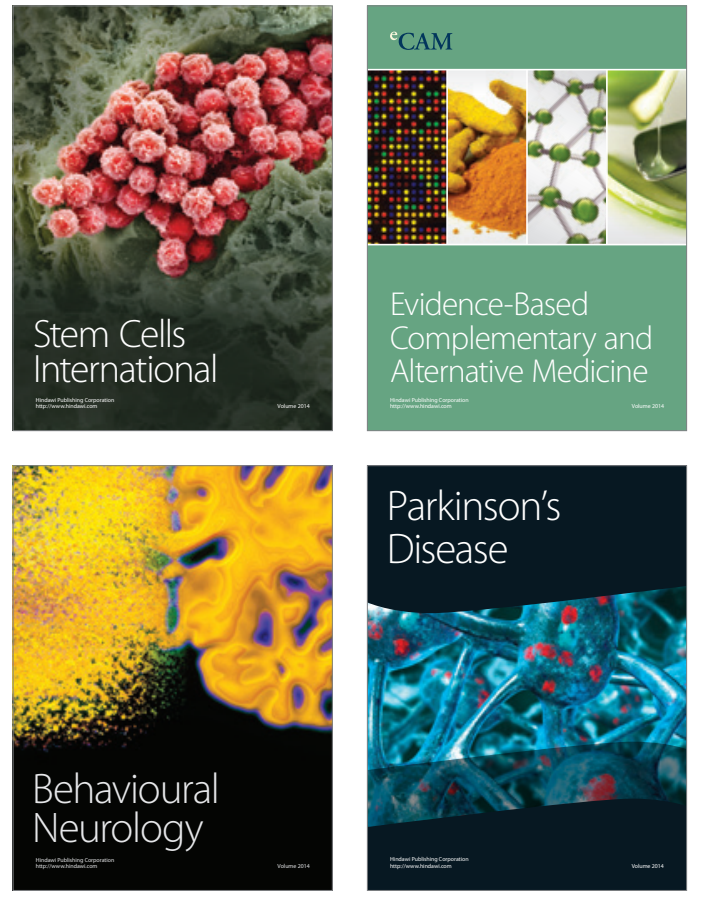
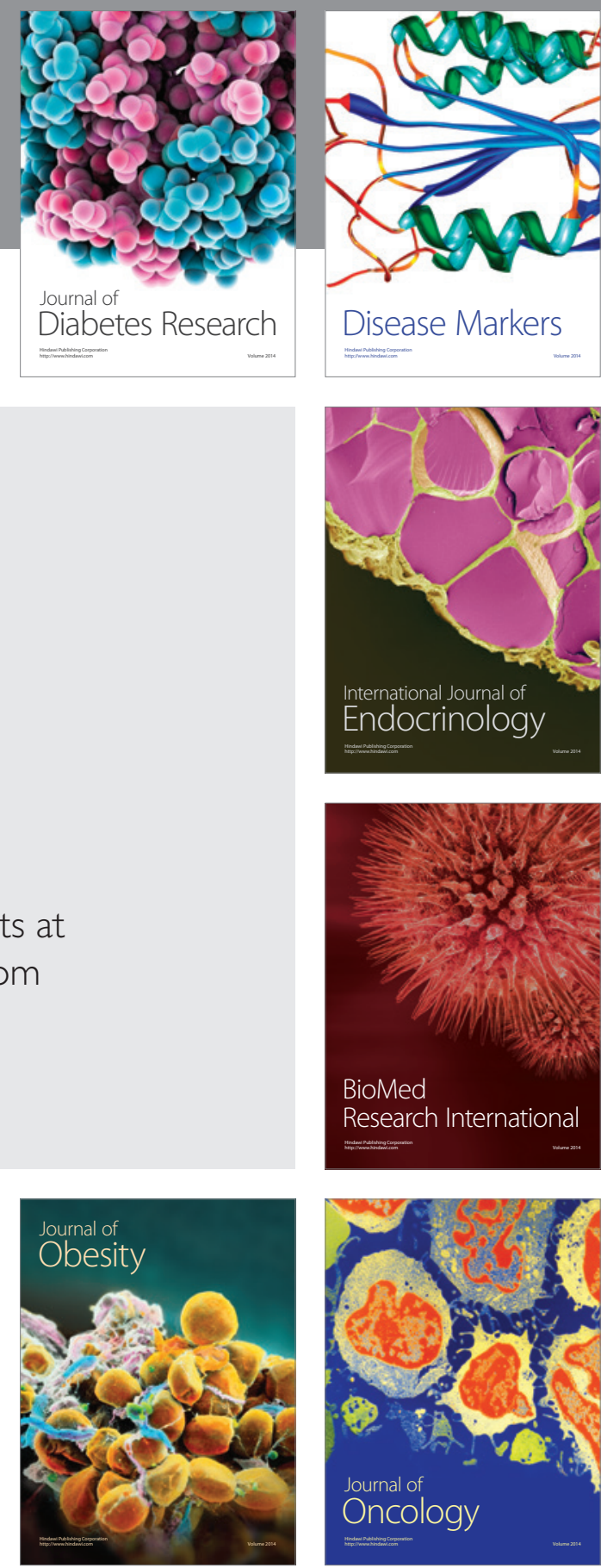

Disease Markers
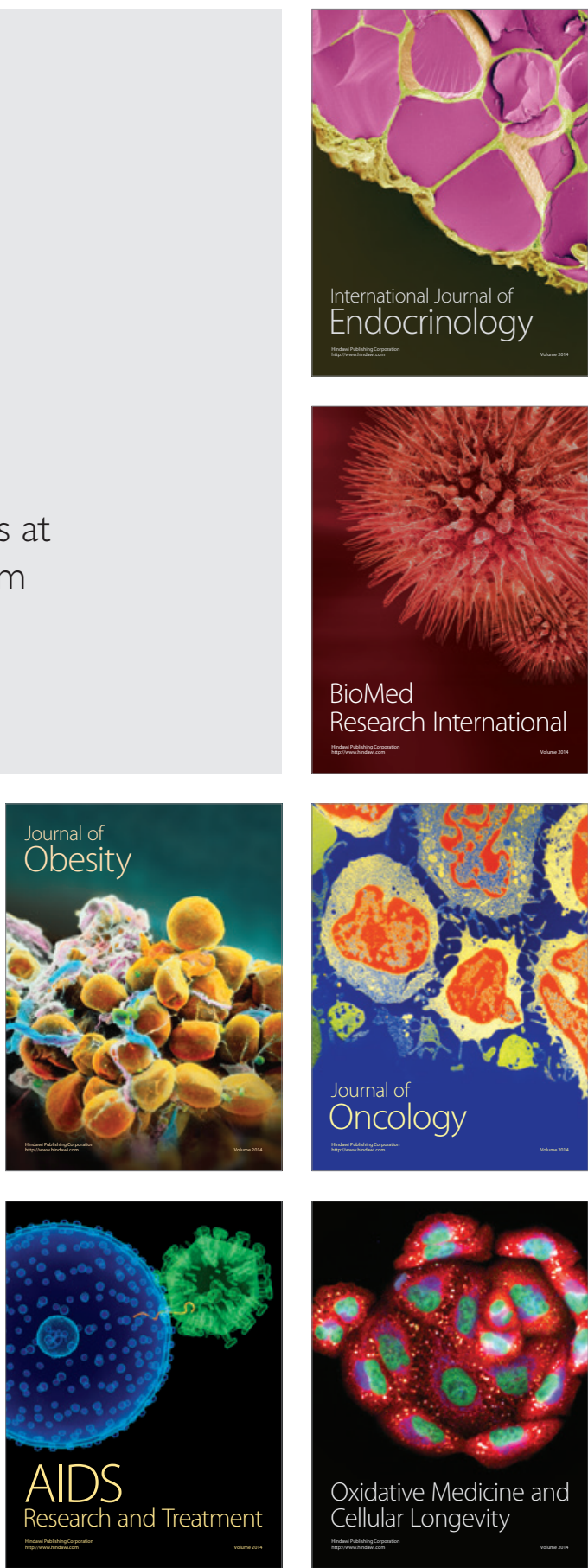